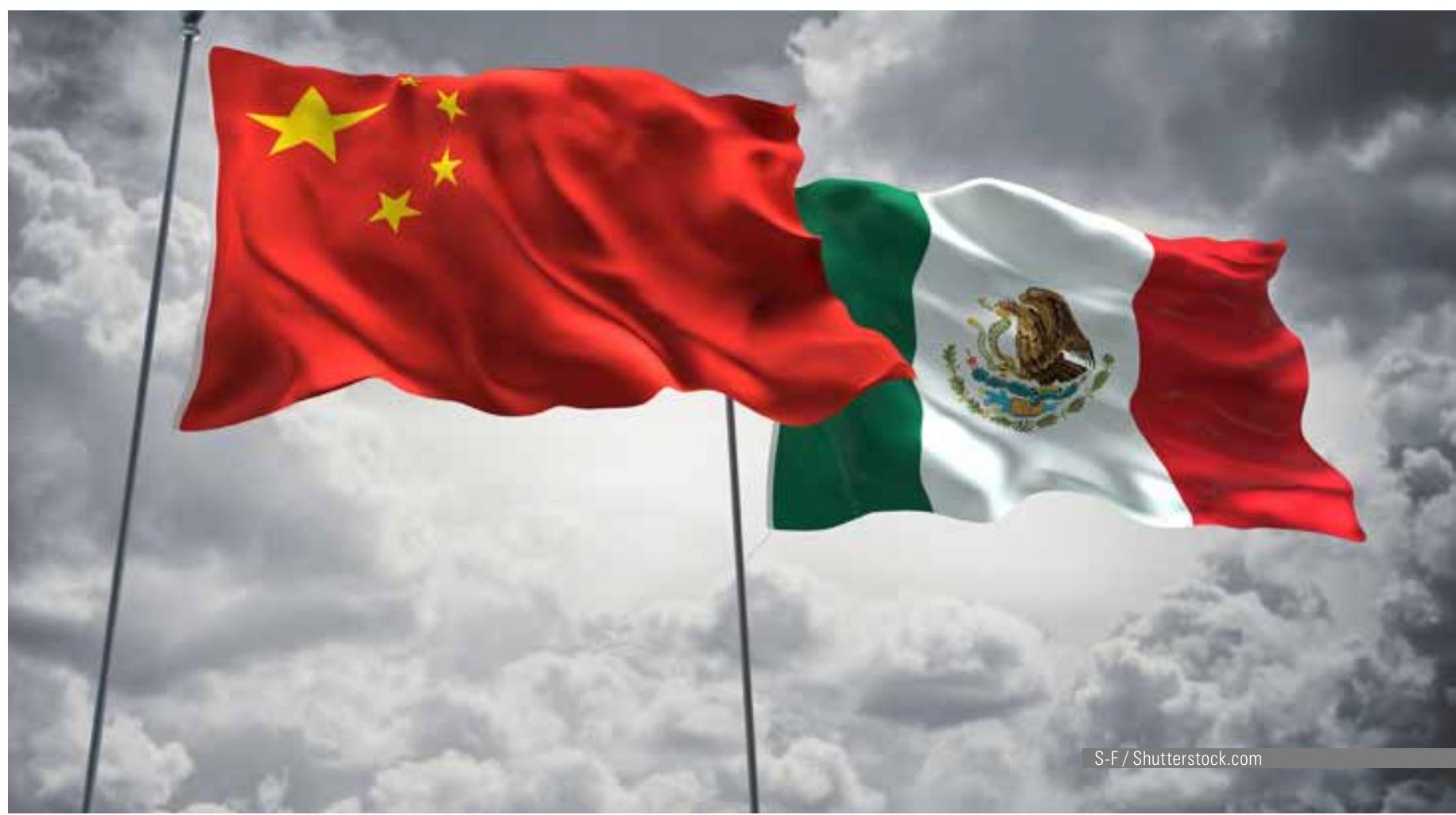

\title{
Ciudades intermedias: un comparativo México-China
}

\section{Omar Neme Castillo}

Profesor-Investigador del Instituto Politécnico Nacional. Escuela Superior de Economía. México oneme@ipn.mx https://orcid.org/0000-0001-8509-7937.

\section{Ana Lilia Valderrama Santibáñez} Profesor-Investigador del Instituto Politécnico Nacional. Escuela Superior de Economía. México avalderrama@ipn.mx

\section{José Israel Briseño Perezyera}

Profesor-Investigador del Instituto

Politécnico Nacional. Escuela Superior de Economía. México

jobriper@hotmail.com

Recibido: 11 de julio 2019

Aprobado: 22 de septiembre 2019

Publicado: 13 de diciembre 2019

\section{Resumen}

Desde sus reformas económicas, México y China han registrado rápidas expansiones urbanas que modificaron la distribución de sus sistemas urbanísticos, llevando a un mayor peso relativo de las ciudades intermedias. El documento tiene como objetivo examinar la dispersión del tamaño de ciudades para ambas economías en 2005 y 2010 , respectivamente. Se emplea la ecuación de rango-tamaño, original y ajustada, y se sigue una metodología econométrica de Mínimos Cuadrados Ordinarios de rangos móviles para estimar el coeficiente de Pareto. El tamaño se define en función del PIB de cada ciudad de al menos 15.000 habitantes, esto representa un aspecto original del documento, pues considera esa dimensión de la urbe desde una perspectiva diferente al de la población. La hipótesis es un exponente Pareto inferior a la unidad. Las estimaciones por submuestras sugieren la existencia de una distribución del tamaño de las ciudades relativamente equitativa en ambas naciones. Como las principales ciudades intermedias se agrupan en sistemas urbanos comparativamente equitativos, se acepta que la desviación del exponente se explica por el mayor peso económico relativo de las ciudades intermedias. La principal limitación es la disponibilidad de datos que significa una disparidad de años entre las economías.

\section{Palabras Clave}

Ciudades intermedias, Exponente de Pareto, Ley de Zipf, PIB urbano, México. China. 


\section{Abstract}

Since their economic reforms, Mexico and China have registered a rapid urban expansion that modified the distribution of the urban system leading to a higher relative weight of the intermediate cities. This paper aims to examine the cities' size dispersion for both economies in 2005 and 2010, respectively. The original and adjusted range-size equation is used in addition to an econometric methodology of Ordinary Least Squares of mobile ranges to estimate the Pareto coefficient. The size is defined according to each city's GDP of at least 15, 000 inhabitants, instead of using the population as a measure of size, representing an original aspect of the paper. The hypothesis is a Pareto exponent lower than the unit. Estimates by subsamples suggest the existence of relatively equal size distribution of cities in both nations. As the main intermediate cities are grouped into comparatively equitable urban systems, it is accepted that the higher relative economic weight of intermediate cities explains the deviation of the exponent. The main limitation is the availability of data which means a disparity of years between economies.

\section{Key words}

Intermediate cities, Pareto distribution, Zipf's law, urban GDP. Mexico. China.

\section{Introducción}

La urbanización es un fenómeno implícito que acompaña al crecimiento económico dado que en las ciudades se concentran procesos industriales, de generación del conocimiento, de inversión y de innovación. Es un proceso complejo a través del cual las comunidades de un país se hacen más grandes, especializadas y eficientes (Fang et al., 2017). Esto es especialmente cierto para países en desarrollo que han experimentado transformaciones socioeconómicas desde el sector agrícola hacia economías dominadas por la industria y, en algún grado, por los servicios.

México y China son naciones que se caracterizan por este tipo de proceso. En las últimas décadas, las ciudades intermedias - aquellas entre 500 mil y un millón de habitantes- muestran una creciente importancia en la economía nacional de cada país, lo que es evidente al considerar su papel dentro de la red de ciudades. Con datos al 2010, este criterio poblacional lo cumplen 32 ciudades en México y 132 en China. La población total en este grupo de ciudades es de 21,9 millones, para concentrar 22,4\% de la población urbana en México. En China, la población total es de 92,9 millones, representando $12,5 \%$ de la población urbana. Al mismo tiempo, contribuyen respectivamente con $23,9 \%$ y $19,1 \%$ del Producto Interno Bruto (PIB) nacional. En consecuencia, la importancia de la producción de las ciudades intermedias en el producto agregado es más proporcional que la de su población, sugiriendo una distorsión en la distribución del tamaño de ciudades que, en última instancia, condiciona el desempeño económico agregado y per cápita en ambos países.

Desde las reformas económicas caracterizadas por la apertura del mercado doméstico, las dos naciones registraron crecimientos importantes, con tasas anuales promedio de 9,5\% en China y 2,4\% en México en los últimos 35 años. Simultáneamente, han evidenciado una rápida expansión urbana que modificó la composición geográfica de la población. En la década de 1980 solo 66,3\% de la población vivía en zonas urbanas en México y 19,4\% en China. En contraste, en 2015 la población urbana representó $79,2 \%$ y 55,6\% del total para cada uno. Este valor es superior al promedio mundial $(53,8 \%)$, aunque por debajo del promedio de los países desarrollados $(81,2 \%)$. 
Una consecuencia central de la urbanización es el cambio en la distribución del tamaño de ciudades, esto es, del número de personas residiendo en una ciudad determinada. La distribución del tamaño de las ciudades se describe tanto por el número como por el tamaño de ciudades individuales (Veneri, 2016). En particular, en esos mismos años la población en urbes intermedias pasó de 6,2 a 16,3\% del total en México y de 11,4 a 13,5\% en China, para representar la cuarta categoría de importancia en el país latinoamericano y la tercera en el asiático. Asimismo, el número de ciudades intermedias creció a una tasa anual de 4,2\% en México, llegando a 21, y en China a tasa de 4,8\% anual, para alcanzar las 155 ciudades en 2015. Para ambos países fue la categoría de ciudad con mayor dinámica.

Una de las regularidades más importantes respecto a la ubicación geográfica de la actividad económica en los países es la concentración en las ciudades. Krugman (1996) observa que esta regularidad distributiva de las aglomeraciones urbanas es una cuasi-ley universal. Puesto que las ciudades tienen tamaños muy variados, existe una tradición empírica urbana (Rosen y Resnick, 1980; Parr y Jones, 1983) que describe el tamaño de la distribución de las ciudades al interior de los sistemas urbanos. Esta literatura se caracteriza por aplicar la distribución de Pareto a la distribución del tamaño de las ciudades de un país, y consideran un caso particular de esta distribución conocido como la regla rango-tamaño (Gabaix y Loannides, 2004). Esta regla establece que el producto del rango y población de una ciudad es aproximadamente constante. De acuerdo con Soo (2005), cuando las ciudades se ordenan por el tamaño de su población y se regresa el logaritmo de su rango en función del logaritmo de su tamaño se genera el coeficiente - exponente de Pareto-, interpretado como una medida de la concentración de la población de las ciudades de diferentes tamaños. Cuando este exponente es igual a uno satisface las condiciones la Ley de Zipf. Matemáticamente, esto significa que la probabilidad de que el tamaño de una ciudad sea mayor a cierta $S$ es $P(s>S)=\alpha / S \xi$, con $\xi \approx 1$ y $\alpha$ una constante positiva (Gabaix, 1999). En este sentido Gabaix (1999) afirma que uno de los hechos estilizados acerca del tamaño relativo de las ciudades en economías de mercado es el cumplimiento de la Ley de Zipf.

De este modo, a pesar de que parece aceptarse la relación natural entre los procesos de urbanización y crecimiento económico, la evolución de la distribución del tamaño de las ciudades en el proceso de desarrollo económico dista mucho de ser directa. Por ende, puede preguntarse si una distribución del tamaño de las ciudades más equitativa favorece o perjudica el desempeño económico de un país. En este sentido, tradicionalmente la relación negativa de la Ley de Pareto entre los logaritmos del tamaño de la ciudad y el rango de la ciudad se considera en función del número de habitantes o la extensión de la tierra; sin embargo, en este documento el tamaño de la ciudad se define al considerar la magnitud de su economía ligada al tamaño de su población, esto es, el tamaño está determinado directamente por el Producto Interno Bruto. Esta alternativa facilita identificar, ceteris paribus, a las ciudades con capacidad de producción saturada, dada su elevada población, y aquellas con más posibilidades de producción al considerar su nivel de población medio.

Por tanto, se mide la distribución del tamaño de las ciudades por el coeficiente de Pareto obtenido de una regresión auxiliar generalmente aceptada en la literatura. Para ello, primero se ordenan las ciudades por tamaño del PIB y se registra su ranking. Después se regresan los logaritmos de estos rangos 
con sus correspondientes tamaños en logaritmos, siguiendo la metodología de Mínimos Cuadrados Ordinarios (MCO). Formalmente:

$$
\ln (\text { Rango })=\alpha_{0}-\alpha_{1} \ln (\text { Tamaño })+\text { Error }
$$

El coeficiente estimado asociado con el logaritmo del tamaño ( $\alpha 1)$ es un estadístico que representa la distribución de tamaño de las ciudades. De acuerdo con Arshad et al. (2018), cuando el exponente de Pareto es igual a uno, se cumple la Ley de Zipf. Entre mayor sea $\alpha 1$, más equitativa es la distribución en el sistema urbano. Por el contrario, menores valores del exponente señalan un sistema de ciudades más desigual con predominio de una o pocas ciudades. En el extremo, cuando $\alpha 1=\infty$, el sistema urbano muestra el mayor grado de igualdad con todas las ciudades aproximadamente del mismo tamaño. Cuando el $\alpha 1=0$, el sistema urbano tiene una distribución desigual con una o pocas ciudades dominantes.

En este sentido, Berry y Okulicz (2012) señalan que la distribución del tamaño de las ciudades con coeficiente de Pareto igual a uno puede considerarse como la distribución óptima que se observaría en la ausencia de fricciones u obstáculos al funcionamiento normal de la economía al interior de un país. La existencia de distorsiones genera que ciudades de menor rango tengan mayor peso en el desempeño económico agregado, compensando en algún grado el menor dinamismo de las ciudades más grandes.

Así, el documento tiene como objetivo estimar el coeficiente de Pareto, esto es, examinar la posible dispersión del tamaño de ciudades. Se establece como hipótesis un coeficiente Pareto menor a la unidad, lo que sería consecuencia del mayor peso económico relativo de las ciudades intermedias. En otras palabras, el comportamiento de las ciudades intermedias representaría un mecanismo subyacente que impulsa el desempeño económico en estas dos naciones. Aún más, reconociendo la heterogeneidad en el tamaño de las economías y de las poblaciones en los países estudiados —el PIB de China es 7,7 veces el de México y la población 10,5 veces-, se espera que el efecto estimado sea más elevado en México que en el país asiático, dada la mayor importancia relativa de las ciudades intermedias en la red de ciudades en la nación.

Dada la disponibilidad de datos, el análisis se realiza para 2010 en China y 2005 en México e incluye 132 ciudades en el primero y 32 en el segundo, que pueden considerarse como ciudades intermedias de acuerdo con la definición del ONU-Habitat (2012). El resto del documento se organiza como sigue: se identifican brevemente algunas regularidades empíricas en las ciudades intermedias y similitudes entre estas en ambos países; se describen la metodología y los datos para plantear un modelo que examine el efecto del coeficiente de Pareto y se discuten los resultados, y, finalmente, se presentan algunas reflexiones. 


\section{Regularidades empíricas en las ciudades intermedias}

La literatura reconoce que la urbanización surge de distintos factores de naturaleza económica (crecimiento, empleo, etc.), demográfica, educativa, tecnológica, ambiental y de infraestructura (Rani y Tripathi, 2016; Hofmann y Wan, 2013). En particular, la urbanización genera más oportunidades de empleo al tiempo que atrae mano de obra desde las zonas rurales, impactando en la dimensión socioeconómica de ambos contextos y, en última instancia, en el desempeño económico.

Así, desde las reformas económicas implementadas en la década de 1980, ambos países han pasado por procesos de urbanización, si bien más intenso en China que en México. Esto puede observarse en la Tabla 1.

Tabla 1. Población urbana en México y China (unidades en miles de personas). ${ }^{1}$

\begin{tabular}{|c|c|c|c|c|c|c|c|c|c|c|c|c|}
\hline \multirow[b]{2}{*}{ Año } & \multicolumn{6}{|c|}{ México } & \multicolumn{6}{|c|}{ China } \\
\hline & $\begin{array}{c}\text { Población } \\
\text { total (miles } \\
\text { de personas) }\end{array}$ & $\begin{array}{c}\text { Crecimiento } \\
\text { anual } \\
\text { promedio } \\
(\%) \\
\end{array}$ & $\begin{array}{c}\text { Población } \\
\text { urbana (miles } \\
\text { de personas) }\end{array}$ & $\begin{array}{c}\text { Crecimiento } \\
\text { anual } \\
\text { promedio } \\
(\%) \\
\end{array}$ & $\begin{array}{c}\text { Participación } \\
(\%)\end{array}$ & $\begin{array}{l}\text { Número de } \\
\text { ciudades }\end{array}$ & $\begin{array}{c}\text { Población } \\
\text { total (miles } \\
\text { de personas) }\end{array}$ & $\begin{array}{c}\text { Crecimiento } \\
\text { anual } \\
\text { promedio } \\
(\%) \\
\end{array}$ & $\begin{array}{c}\text { Población } \\
\text { urbana (miles } \\
\text { de personas) }\end{array}$ & $\begin{array}{c}\text { Crecimiento } \\
\text { anual } \\
\text { promedio } \\
(\%) \\
\end{array}$ & $\begin{array}{c}\text { Participación } \\
(\%)\end{array}$ & $\begin{array}{l}\text { Número de } \\
\text { ciudades }\end{array}$ \\
\hline 1950 & 28,296 & - & 12,069 & - & 42.65 & 3 & 543,776 & - & 64,180 & - & 11.80 & 34 \\
\hline 1955 & 32,906 & 3.02 & 15,360 & 5.21 & 46.68 & 4 & 600,995 & 2.00 & 83,276 & 4.82 & 13.86 & 39 \\
\hline 1960 & 38,677 & 3.23 & 19,630 & 4.72 & 50.75 & 4 & 650,680 & 1.59 & 105,427 & 4.90 & 16.20 & 50 \\
\hline 1965 & 45,339 & 3.18 & 24,905 & 3.99 & 54.93 & 7 & 711,547 & 1.79 & 128,692 & 4.76 & 18.09 & 56 \\
\hline 1970 & 52,988 & 3.12 & 31,274 & 1.93 & 59.02 & 9 & 814,378 & 2.70 & 141,702 & 4.55 & 17.40 & 64 \\
\hline 1975 & 61,708 & 3.05 & 38,728 & 2.30 & 62.76 & 15 & 913,570 & 2.30 & 158,961 & 4.28 & 17.40 & 80 \\
\hline 1980 & 70,353 & 2.62 & 46,672 & 3.62 & 66.34 & 17 & 984,016 & 1.49 & 190,483 & 3.73 & 19.36 & 90 \\
\hline 1985 & 77,859 & 2.03 & 53,684 & 4.87 & 68.95 & 26 & $1,062,299$ & 1.53 & 242,987 & 2.80 & 22.87 & 105 \\
\hline 1990 & 86,077 & 2.01 & 61,475 & 4.75 & 71.42 & 35 & $1,165,429$ & 1.85 & 308,167 & 2.71 & 26.44 & 136 \\
\hline 1995 & 95,393 & 2.06 & 69,987 & 4.36 & 73.37 & 39 & $1,237,531$ & 1.20 & 383,156 & 2.59 & 30.96 & 179 \\
\hline 2000 & 103,874 & 1.70 & 77,617 & 3.63 & 74.72 & 43 & $1,280,429$ & 0.68 & 459,383 & 2.07 & 35.88 & 264 \\
\hline 2005 & 110,732 & 1.28 & 84,498 & 3.98 & 76.31 & 46 & $1,318,177$ & 0.58 & 560,518 & 1.70 & 42.52 & 322 \\
\hline 2010 & 117,886 & 1.25 & 91,745 & 3.55 & 77.83 & 49 & $1,359,821$ & 0.62 & 669,386 & 1.65 & 49.23 & 362 \\
\hline 2015 & 125,236 & 1.21 & 99,245 & 3.05 & 79.25 & 52 & $1,401,587$ & 0.61 & 779,479 & 1.57 & 55.61 & 407 \\
\hline 2020 & 131,955 & 1.05 & 106,298 & 2.30 & 80.56 & 55 & $1,432,868$ & 0.44 & 874,427 & 1.37 & 61.03 & 444 \\
\hline 2025 & 138,195 & 0.92 & 112,981 & 1.61 & 81.76 & 58 & $1,448,984$ & 0.22 & 947,540 & 1.22 & 65.39 & 484 \\
\hline 2030 & 143,663 & 0.78 & 119,016 & 1.06 & 82.84 & 60 & $1,453,297$ & 0.06 & 998,925 & 1.04 & 68.74 & 504 \\
\hline
\end{tabular}

Fuente: elaboración propia con datos de United Nations, Department of Economic and Social Affairs, Population Division (2014). World Urbanization Prospects: The 2014 Revision.

La población total en China creció 1,42 veces en 2015 frente a 1980, pasando de 984 millones hasta 1.401,6 millones de personas, al tiempo que la población urbana creció 4,09 veces, desde 190,5 millones hasta 779,5 millones de habitantes. Así, la tasa de crecimiento promedio anual de la población urbana fue mayor a la total (1,02\% y $4,1 \%$, respectivamente). En contraste, la población total en México pasó de 70,4 millones en 1980 a 125,3 millones en 2015, siendo 1,78 veces más grande; simultáneamente, la población urbana creció 2,12, desde 46,6 millones hasta 99,3 millones de habitantes. Las

1 México cuenta con 32 entidades federativas, 2.457 municipios y 16 alcaldías (Ciudad de México CDMX -). China tiene 34 unidades administrativas de nivel provincial, incluyendo 4 municipios bajo jurisdicción central (Beijing, Shanghai, Tianjin y Chongqing), 23 provincias (Hebei, Shanxi, Liaoning, Jilin Heilongjiang, Jiansu, Zhejiang, Anhui, Fujian, Jiangxi, Shandong, Henan, Hubei, Hunan, Guangdong, Hainan, Sichuan, Guizhou, Yunnan, Shaanxi, Gansu, Qinghai y Taiwán), cinco regiones autónomas (Mongolia Interior, Guangxi, Ningxia, Tibet, Xinjiang) y dos regiones administrativas especiales (Honk Kong y Macao). Ciudades con 300.000 o más habitantes. 
poblaciones total y urbana registraron tasas de crecimiento promedio anual de $1,7 \%$ y $2,2 \%$, cada una.

En la década 1980, al inicio de las reformas económicas, China tenía 90 ciudades de al menos 300.000 habitantes, para alcanzar 407 ciudades de esta envergadura en 2015, lo que representa un crecimiento de $352,2 \%$. En ese mismo periodo, el número de ciudades con ese criterio en México pasó de 17 a 52, con un ritmo de crecimiento del 205,9\%. Al respecto, China implementó un esquema para el desarrollo de áreas urbanas, basado en la idea de un estricto control de las ciudades de mayor tamaño, desarrollo razonable de ciudades medianas y un agresivo desarrollo de ciudades menores. Estas políticas urbanas llevaron a que las áreas urbanas medianas y pequeñas crecieran rápidamente y al desarrollo de ciudades-satélite cercanas a las ciudades grandes (Lin et al., 2013)

En el caso mexicano, el perfil urbano e industrial al inicio de la década de 1980 condujo a una nueva etapa en el proceso de urbanización, caracterizada por seis elementos: i) disminución del peso relativo de la Zona Metropolitana de la Ciudad de México frente al resto del sistema urbano nacional; ii) descenso demográfico de las cuatro zonas metropolitanas más importantes (México, Guadalajara, Monterrey y Puebla; iii) movimientos demográficos intrametropolitanos tipo centro-periferia; iv) reorientación de flujos migratorios interurbanos e interregionales; v) crecimiento relativo más importante en ciudades de menor tamaño; y, vi) redistribución de la población sobre el territorio (Sobrino, 1999).

En este sentido, la rápida urbanización puede apreciarse en el incremento del número de ciudades. En la Tabla 2 se muestra el porcentaje de población urbana y el número de aglomeraciones de acuerdo a seis categorías del tamaño poblacional propuesta por la División de Población de la ONU, a saber: i) 10 millones o más; ii) 5 a 10 millones; iii) 1 a 5 millones; iv) 500.000 a 1 millón; v) 300.000 a 500.000; y, vi) menos de 300.000 habitantes.

En general, se observa un incremento de 1980 a 2015 en el número total de ciudades. En particular, para México, la tasa de aumento en el número de ciudades intermedias fue de 320\%; al mismo tiempo, la población en estas aglomeraciones representa en el último año 16,3\% del total de la población urbana, para registrar un crecimiento promedio anual de 2,8\%. En China, 155 ciudades llegaron a este rango en 2015, lo que implica que la tasa de incremento fue de $416,7 \%$, mayor a la mexicana. En contraste, el porcentaje de población en este tipo de aglomeraciones en el total de población urbana creció a tasa anual de 0,48\% —-menor que en México-, representando $13,5 \%$ del total en 2010.

En ambos países esta categoría experimentó avances. Considerando el peso relativo en la población urbana total, se observa que en México se presenta mayor crecimiento por arriba de las ciudades entre 1 y 5 millones y de 10 millones o más; y en China es la tercera en el ritmo de crecimiento, por debajo de estas dos mismas categorías. Respecto al número de aglomeraciones urbanas, en México es la segunda de mayor avance (debajo de ciudades entre 1 y 5 millones) y en China es la de mayor crecimiento. Estos datos indican que los dos países han experimentado un notable crecimiento en las ciudades intermedias, con dinámicas por arriba del promedio, ganando, en consecuencia, importancia relativa en el sistema de centros urbanos en cada país. 
Tabla 2. Porcentaje de población urbana y número de aglomeraciones, por tamaño de ciudad.

\begin{tabular}{|c|c|c|c|c|c|c|c|c|c|c|c|c|}
\hline \multicolumn{13}{|c|}{ Porcentaje de población urbana } \\
\hline Tipo de ciudad & 1980 & 1985 & 1990 & 1995 & 2000 & 2005 & 2010 & 2015 & 2020 & 2025 & 2030 & $\begin{array}{c}\text { Crecimiento Anual } \\
\text { Promedio } \\
1980-2015(\%)\end{array}$ \\
\hline \multicolumn{13}{|l|}{ Mexico } \\
\hline 10 millones o más & 27.9 & 26.6 & 25.4 & 24.3 & 23.8 & 22.8 & 21.9 & 21.2 & 20.6 & 20.3 & 20.1 & -0.788 \\
\hline 5 a 10 milliones & 0 & 0 & 0 & 0 & 0 & 0 & 0 & 0 & 4.9 & 9.5 & 9.5 & - \\
\hline 1 a 5 millones & 11.4 & 11.8 & 14.0 & 18.5 & 20.4 & 20.6 & 23.2 & 26.8 & 25.1 & 26.3 & 28.3 & 2.458 \\
\hline 500,000 a 1 millón & 6.2 & 9.9 & 16.4 & 13.6 & 17.3 & 18.2 & 18.5 & 16.3 & 15.2 & 11.3 & 10.1 & 2.787 \\
\hline 300,000 a 500,000 & 6.6 & 10.1 & 9.4 & 9.7 & 6.8 & 7.2 & 6.1 & 6.6 & 6.1 & 5.7 & 5.9 & -0.009 \\
\hline Menos de 300,000 & 47.8 & 41.6 & 34.7 & 34.0 & 31.7 & 31.1 & 30.2 & 29.1 & 28.0 & 26.9 & 26.1 & -1.405 \\
\hline \multicolumn{13}{|l|}{ China } \\
\hline 10 millones o más & 0 & 0 & 0 & 2.7 & 5.3 & 5.3 & 8.6 & 11.8 & 12.1 & 12.2 & 13.1 & - \\
\hline 5 a 10 milliones & 5.9 & 5.3 & 4.7 & 5.1 & 7.6 & 12.0 & 10.3 & 8.6 & 11.0 & 11.6 & 11.6 & 1.056 \\
\hline 1 a 5 millones & 17.7 & 18.3 & 20.8 & 21.8 & 24.8 & 21.1 & 21.4 & 22.9 & 23.2 & 24.2 & 24.6 & 0.732 \\
\hline 500,000 a 1 millón & 11.4 & 10.4 & 8.3 & 9.3 & 11.8 & 12.1 & 12.4 & 13.5 & 13.4 & 13.8 & 13.9 & 0.475 \\
\hline 300,000 a 500,000 & 8.0 & 7.1 & 7.9 & 8.1 & 9.7 & 10.5 & 9.3 & 7.3 & 6.6 & 6.7 & 6.3 & -0.272 \\
\hline Menos de 300,000 & 56.9 & 58.9 & 58.3 & 53.0 & 40.8 & 39.1 & 38.0 & 36.0 & 33.7 & 31.6 & 30.4 & -1.300 \\
\hline \multicolumn{13}{|c|}{ Numero de aglomeraciones } \\
\hline Tipo de ciudad & 1980 & 1985 & 1990 & 1995 & 2000 & 2005 & 2010 & 2015 & 2020 & 2025 & 2030 & $\begin{array}{l}\text { Crecimiento } \\
\text { Promedio } \\
1980-2015(\%) \\
\end{array}$ \\
\hline \multicolumn{13}{|l|}{ Mexico } \\
\hline 10 millones o más & 1 & 1 & 1 & 1 & 1 & 1 & 1 & 1 & 1 & 1 & 1 & 0.00 \\
\hline 5 a 10 milliones & 0 & 0 & 0 & 0 & 0 & 0 & 0 & 0 & 1 & 2 & 2 & - \\
\hline 1 a 5 millones & 3 & 3 & 4 & 7 & 8 & 8 & 10 & 13 & 15 & 20 & 22 & 333.33 \\
\hline 500,000 a 1 millón & 5 & 8 & 15 & 14 & 20 & 21 & 23 & 21 & 21 & 18 & 17 & 320.00 \\
\hline 300,000 a 500,000 & 8 & 14 & 15 & 17 & 14 & 16 & 15 & 17 & 17 & 17 & 18 & 112.50 \\
\hline Total & 17 & 26 & 35 & 39 & 43 & 46 & 49 & 52 & 55 & 58 & 60 & 205.88 \\
\hline \multicolumn{13}{|l|}{ China } \\
\hline 10 millones o más & 0 & 0 & 0 & 1 & 2 & 2 & 4 & 6 & 6 & 6 & 7 & - \\
\hline 5 a 10 milliones & 2 & 2 & 2 & 3 & 5 & 10 & 10 & 10 & 14 & 15 & 16 & 400.00 \\
\hline 1 a 5 millones & 18 & 23 & 34 & 43 & 58 & 60 & 71 & 89 & 105 & 115 & 125 & 394.44 \\
\hline 500,000 a 1 millón & 30 & 35 & 37 & 53 & 81 & 97 & 119 & 155 & 170 & 184 & 194 & 416.67 \\
\hline 300,000 a 500,000 & 40 & 45 & 63 & 79 & 118 & 153 & 158 & 147 & 149 & 164 & 162 & 267.50 \\
\hline Total & 90 & 105 & 136 & 179 & 264 & 322 & 362 & 407 & 444 & 484 & 504 & 352.22 \\
\hline
\end{tabular}

Los datos históricos disponibles para las 16 alcaldías -antes delegaciones- de la Ciudad de México, anteriormente Distrito Federal, se agrupan, por cuestiones administrativas y políticas, en una aglomeración urbana de may or tamaño, esto es, en la Ciudad de México. Fuente: elaboración propia con datos de United Nations, Department of Economic and Social Affairs, Population Division (2014).

World Urbanization Prospects: The 2014 Revision.

Fuente: elaboración propia con datos de United Nations, Department of Economic and Social Affairs, Population Division (2014). World Urbanization Prospects: The 2014 Revision.

Por otro lado, las ciudades intermedias se muestran en las Tablas 3 y 4 , en México y China respectivamente, ordenadas de mayor a menor PIB. Para México se tiene el PIB de 2005 en dólares a precios del 2010 y para China el PIB de 2016 a dólares constantes del 2010. 
Tabla 3. Tamaño de las ciudades intermedias en México (PIB), por orden de importancia

\begin{tabular}{|c|c|c|c|c|c|c|c|c|c|c|c|c|c|}
\hline Rango 1 & Rango 2 & Ciudad & Estado & $\begin{array}{c}\text { Población } \\
(2010)\end{array}$ & PIB (2005) & PIBPC (2005) & Rango 1 & Rango 2 & Ciudad & Estado & $\begin{array}{c}\text { Población } \\
(2010)\end{array}$ & PIB (2005) & PIBPC (2005) \\
\hline 1 & 11 & Mérida & Yucatán & 830,732 & $13,545,234$ & $16,981.0$ & 17 & 30 & Toluca & México & 819,561 & $9,330,837$ & $11,857.1$ \\
\hline 2 & 12 & Chihuahua & Chihuahua & 819,543 & $13,350,344$ & $16,965.2$ & 18 & 31 & Cuautitlán Izcalli & México & 511,675 & $8,656,337$ & $17,618.9$ \\
\hline 3 & 13 & Coyoacán & Ciudad de México & 620,416 & $13,231,487$ & $22,210.8$ & 19 & 32 & Cuauhtémoc & Ciudad de México & 531,831 & $8,417,872$ & $16,484.2$ \\
\hline 4 & 14 & San Luis Potosí & San Luis Potosí & 772,604 & $13,083,418$ & $17,636.1$ & 20 & 34 & Veracruz & Veracruz & 552,156 & $8,130,838$ & $15,336.0$ \\
\hline 5 & 15 & Querétaro & Querétaro & 801,940 & $12,590,897$ & $16,351.3$ & 21 & 36 & Saltillo & Coahuila & 725,123 & $7,304,649$ & $10,491.2$ \\
\hline 6 & 16 & Naucalpan & México & 833,779 & $12,433,997$ & $15,531.0$ & 22 & 37 & Torreón & Coahuila & 639,629 & $7,270,567$ & $11,838.0$ \\
\hline 7 & 18 & Hermosillo & Sonora & 784,342 & $11,476,793$ & $15,238.9$ & 23 & 38 & Reynosa & Tamaulipas & 608,891 & $7,158,564$ & $12,244.0$ \\
\hline 8 & 19 & Mexicali & Baja California & 936,826 & $11,439,485$ & $12,717.0$ & 24 & 42 & Tlaquepaque & Jalisco & 608,114 & $6,527,897$ & $11,179.6$ \\
\hline 9 & 20 & Guadalupe & Nuevo León & 678,006 & $10,787,528$ & $16,570.2$ & 25 & 43 & Centro & Tabasco & 640,359 & $6,356,483$ & $10,337.9$ \\
\hline 10 & 21 & Tlalnepantla & México & 664,225 & $10,738,077$ & $16,836.4$ & 26 & 47 & Acapulco & Guerrero & 789,971 & $5,825,240$ & $7,679.6$ \\
\hline 11 & 23 & Benito Juárez & Quintana Roo & 661,176 & $10,439,514$ & $16,443.8$ & 27 & 48 & Apodaca & Nuevo León & 523,370 & $5,708,686$ & $11,359.7$ \\
\hline 12 & 24 & Culiacán & Sinaloa & 858,638 & $10,416,901$ & $12,634.8$ & 28 & 49 & Tuxtla Gutiérrez & Chiapas & 553,374 & $5,645,745$ & $10,625.3$ \\
\hline 13 & 25 & Álvaro Obregón & Ciudad de México & 727,034 & $10,302,536$ & $14,758.0$ & 29 & 50 & Tultitlán & México & 524,074 & $5,611,276$ & $11,150.8$ \\
\hline 14 & 26 & Tlalpan & Ciudad de México & 650,567 & $9,977,413$ & $15,972.2$ & 30 & 54 & Durango & Durango & 582,267 & $5,225,058$ & $9,345.6$ \\
\hline 15 & 27 & Morelia & Michoacán & 729,279 & $9,628,533$ & $13,750.1$ & 31 & 56 & Irapuato & Guanajuato & 529,440 & $4,962,816$ & $9,762.2$ \\
\hline 16 & 29 & Aguascalientes & Aguascalientes & 797,010 & $9,416,279$ & $12,304.2$ & 32 & 70 & Chimalhuacán & México & 614,453 & $3,980,596$ & $6,746.8$ \\
\hline
\end{tabular}

Rango 1: rango respecto a las ciudades intermedias; Rango 2: rango respecto al total de ciudades.

PIB y PIB PC en dólares constantes al 2010.

Fuente: INEGI. Censo de Población y Vivienda 2010.

Fuente: INEGI. Censo de Población y Vivienda 2010.

Existe un total de 32 ciudades intermedias distribuidas en 22 entidades federativas. La población de estas aglomeraciones oscila entre 936.000 (Mexicali, Baja California) y 511.000 habitantes (Cuautitlán Izcalli, Edomex) La ciudad de mayor tamaño, es decir, peso económico, es Mérida, Yucatán (13,5 miles de millones de dólares — mmd-) y la de menor tamaño Chimalhuacán, Edomex (3,9 mmd). Asimismo, la que registra el mayor nivel de desarrollo —-medido por el PIB per cápita— es Coyoacán (CDMX) con 22.210 dólares y la de menor PIBPC es Chimalhuacán (Edomex) (6.746 dólares). Por el nivel de PIB, las primeras diez ciudades intermedias se ubican dentro de las veinticinco ciudades más importantes del país — dentro del primer cuartil-. Solo cuatro entidades registran dos o más ciudades en esta categoría (México cinco, CDMX cuatro, Coahuila dos y Nuevo León dos). De estas, con la excepción de Saltillo (Coahuila), todas son ciudades que hacen parte de áreas urbanas mayores.

Para China, el total de ciudades intermedias es 132, distribuidas en 22 unidades administrativas, lo que habla de una dispersión similar a México. La población de estas aglomeraciones oscila entre 999.000 (Zhuzhou, Hunan) y 500.000 (Danyang, Jiangsu) y 511.000 habitantes, también similar a la mexicana. La ciudad de mayor tamaño - mayor peso económico- es Ordos, Inner Mongolia (30,5 miles de millones de dólares —-mmd-) y la de menor tamaño Qitaihe, Heilongjiang (3,9 mmd). Asimismo, la que registra el mayor nivel de desarrollo —-medido por el PIB per cápita — es Ordos (Inner Mongolia) con 58.861 dólares y la de menor PIBPC es Qitaihe (Heilongjiang) (2.923 dólares). Por el nivel de PIB, las primeras diez ciudades intermedias se ubican entre las sesenta ciudades más importantes del país — dentro del primer cuartil_. A diferencia de México, todas las unidades administrativas registran dos o más ciudades en esta categoría, excepto Gansu. 
Tabla 4. Tamaño de las ciudades intermedias en China (PIB), por orden de importancia

\begin{tabular}{|c|c|c|c|c|c|c|c|c|c|c|c|c|c|c|c|c|c|c|c|c|}
\hline Rango 1 & Rango 2 & Ciudad & Provincia & $\begin{array}{l}\text { Población } \\
(2010)\end{array}$ & $\operatorname{PIB}(2016)$ & PIBPC (2005) & Rango 1 & Rango 2 & Ciudad & Provincia & $\begin{array}{l}\text { Población } \\
(2010)\end{array}$ & $\operatorname{PIB}(2016)$ & $\operatorname{IBPC}(2005)$ & Rango 1 & Rango 2 & Ciudad & Provincia & $\begin{array}{c}\begin{array}{c}\text { Población } \\
(2010)\end{array} \\
\end{array}$ & PIB (2016) & PIBPC (2005) \\
\hline 1 & 40 & Ordos & Inner Mongolia & 510,242 & $30,505,314$ & $\begin{array}{ll}58,861.8 \\
\end{array}$ & 45 & 131 & Xinxiang & Henan & 918,078 & $14,962,995$ & $16,046.2$ & 89 & 227 & Neijiang & Sichuan & 586,445 & $8,960,535$ & $15,043.2$ \\
\hline 2 & 43 & Jining & Shandong & 939,034 & $29,703,651$ & $31,143.2$ & 46 & 132 & Zaozhuang & Shandong & 980,893 & $14,794,514$ & $14,849.6$ & 90 & 231 & Yangjiang & Guangdong & 692,540 & $8,774,792$ & $12,474.6$ \\
\hline 3 & 45 & Taizhou & Jiangsu & 676,877 & $28,322,664$ & $41,196.4$ & 47 & 133 & Jujuiang & Jiangxi & 611,321 & $14,528,674$ & $23,398.7$ & 91 & 232 & Changhhi & Shanxi & 653,125 & $8,772,720$ & $13,224.3$ \\
\hline 4 & 46 & Nan'an & Fujan & 718,516 & $28,239,114$ & $38,694.5$ & 48 & 134 & Jiaozıo & Henan & 702,527 & $14,466,530$ & $20,273.8$ & 92 & 235 & Zhoushan & Zhejiang & 542,190 & $8,570,406$ & $15,562.7$ \\
\hline 5 & 48 & Ruian & Zhejiang & 927,383 & $27,210,969$ & $28,888.1$ & 49 & 135 & Zhaoqing & Guangdong & 784,642 & $14,389,885$ & $18,055.9$ & 93 & 237 & Zigong & Sichuan & 666,204 & $8,524,833$ & $12,598.3$ \\
\hline 6 & 50 & Jiaxing & Zhejiang & 762,643 & $26,667,551$ & $34,426.8$ & 50 & 137 & Guilin & Guangxi & 963,629 & $14,188,261$ & $14,496.2$ & 94 & 238 & Mudanjiang & Heilongjiang & 790,623 & $8,501,356$ & $10,586.5$ \\
\hline 7 & 51 & Zhenjiang & Jiangsu & 950,516 & $26,472,141$ & $27,419.8$ & 51 & 139 & Xinyang & Henan & 625,302 & $14,070,877$ & $22,154.7$ & 95 & 241 & Shaoguan & Guangdong & 726,267 & $8,412,973$ & $11,404.8$ \\
\hline 8 & 54 & Changshu & Jiangsu & 929,124 & $25,973,605$ & $27,522.8$ & 52 & 142 & Anyang & Henan & 908,129 & $14,015,638$ & $15,195.0$ & 96 & 246 & Linfen & Shanxi & 571,237 & $8,303,185$ & $14,310.8$ \\
\hline 9 & 59 & Tengzhou & Shandong & 783,473 & $25,509,593$ & $32,056.3$ & 53 & 144 & Taixing & Jiangsu & 553,079 & $13,973,518$ & $24,874.4$ & 97 & 250 & Siping & Jilin & 509,107 & $8,243,112$ & $15,941.0$ \\
\hline 10 & 60 & Jinhua & Zhejiang & 710,597 & $25,443,996$ & $35,253.0$ & 54 & 147 & Shangqiu & Henan & 618,549 & $13,735,297$ & $21,862.4$ & 98 & 256 & Yingkou & Liaoning & 880,412 & $7,983,486$ & $8,927.7$ \\
\hline 11 & 61 & Yiwu & Zhejiang & 878,903 & $24,892,982$ & $27,885.0$ & 55 & 148 & Xingtai & Hebei & 668,765 & $13,642,081$ & $20,083.6$ & 99 & 263 & Lu'an & Anhui & 661,217 & $7,651,359$ & $11,392.8$ \\
\hline 12 & 63 & Zhangzhou & Fujian & 614,700 & $24,038,842$ & $38,502.1$ & 56 & 150 & Tongliao & Inner Mongolia & 540,338 & $13,460,481$ & $24,526.2$ & 100 & 272 & Guiping & Guangxi & 508,212 & $7,472,521$ & $14,476.3$ \\
\hline 13 & 64 & Dongying & Shandong & 773,543 & $24,026,413$ & $30,580.1$ & 57 & 151 & Danyang & Jiangsu & 500,572 & $13,362,431$ & $26,281.7$ & 101 & 273 & Luohe & Henan & 575,956 & $7,470,449$ & $12,770.0$ \\
\hline 14 & 68 & Handan & Hebei & 919,295 & $23,208,178$ & $24,855.4$ & 58 & 152 & Chifeng & Inner Mongolia & 902,285 & $13,349,311$ & $14,566.3$ & 102 & 280 & Meizhou & Guangdong & 612,551 & $7,219,800$ & $11,604.3$ \\
\hline 15 & 71 & Weihai & Shandong & 698,863 & $22,180,033$ & $31,246.7$ & 59 & 153 & Baoji & Shaanxi & 871,940 & $13,341,025$ & $15,063.9$ & 103 & 281 & Xinyu & Jiangxi & 567,820 & $7,154,894$ & $12,405.9$ \\
\hline 16 & 75 & Xintai & Shandong & 672,207 & $21,666,306$ & $31,733.4$ & 60 & 155 & Xiangtan & Hunan & 903,287 & $12,890,133$ & $14,049.7$ & 104 & 283 & Jinzhou & Liaoning & 946,098 & $7,131,417$ & $7,421.2$ \\
\hline 17 & 77 & Nanyang & Henan & 899,899 & $21,508,873$ & $23,532.0$ & 61 & 158 & Mianyang & Sichuan & 967,007 & $12,638,794$ & $12,868.0$ & 105 & 288 & Panzhihua & Sichuan & 631,258 & $7,006,438$ & $10,927.6$ \\
\hline 18 & 78 & Yueyang & Hunan & 924,099 & $21,411,514$ & $22,812.0$ & 62 & 160 & Pingdingshan & Henan & 855,130 & $12,602,197$ & $14,509.4$ & 106 & 291 & Panjin & Liaoning & 857,993 & $6,991,247$ & $8,022.4$ \\
\hline 19 & 82 & Changde & Hunan & 846,308 & $20,395,798$ & $23,727.2$ & 63 & 164 & Rizhao & Shandong & 902,272 & $12,446,146$ & $13,581.0$ & 107 & 292 & Lufeng & Guangdong & 579,527 & $6,985,033$ & $11,866.7$ \\
\hline 20 & 84 & Dezhou & Shandong & 526,232 & $20,252,175$ & $37,890.4$ & 64 & 168 & Qujing & Yunnan & 646,046 & $12,210,688$ & $18,608.5$ & 108 & 293 & Suining & Sichuan & 549,826 & $6,962,937$ & 12,468.1 \\
\hline 21 & 85 & Yixing & Jiangsu & 710,497 & $20,207,984$ & $28,002.4$ & 65 & 170 & Kaifeng & Henan & 894,142 & $12,118,852$ & $13,344.1$ & 109 & 295 & Pingxiang & Jiangxi & 716,229 & $6,917,364$ & $9,508.7$ \\
\hline 22 & 89 & Liaocheng & Shandong & 606,366 & $19,742,591$ & $32,055.6$ & 66 & 171 & Deyang & Sichuan & 530,122 & $12,100,899$ & $22,473.8$ & 110 & 304 & Guigang & Guangxi & 658,887 & $6,620,452$ & $9,892.6$ \\
\hline 23 & 92 & Zhangjiagang & Jiangsu & 762,625 & $19,081,098$ & $24,633.5$ & 67 & 172 & Jingzhou & Hubei & 904,157 & $11,923,442$ & $12,983.5$ & 111 & 305 & Tongling & Anhui & 526,261 & $6,609,404$ & $12,365.0$ \\
\hline 24 & 93 & Langfang & Hebei & 530,840 & $18,784,876$ & $34,840.1$ & 68 & 175 & Yibin & Sichuan & 549,650 & $11,414,549$ & $20,445.9$ & 112 & 309 & Xiantao & Hubei & 553,029 & $6,532,759$ & $11,630.1$ \\
\hline 25 & 94 & Wenling & Zhejiang & 749,013 & $18,700,636$ & $24,581.1$ & 69 & 177 & Nanchong & Sichuan & 890,402 & $11,402,810$ & 12,608.4 & 113 & 311 & Beiliu & Guangxi & 652,853 & $6,447,829$ & $9,723.7$ \\
\hline 26 & 97 & Pizhou & Jiangsu & 631,572 & $18,568,752$ & $28,946.4$ & 70 & 181 & Xiaogan & Hubei & 582,403 & $10,887,011$ & $18,404.3$ & 114 & 338 & Jiamusi & Heilongjiang & 631,357 & $5,834,670$ & $9,098.6$ \\
\hline 27 & 98 & Yueqing & Zhejiang & 725,972 & $18,249,053$ & $24,748.8$ & 71 & 183 & Yongzhou & Hunan & 540,930 & $10,849,725$ & $19,747.5$ & 115 & 341 & Tianmen & Hubei & 612,515 & $5,804,289$ & $9,329.7$ \\
\hline 28 & 102 & Heze & Shandong & 559,636 & $17,678,015$ & $31,100.1$ & 72 & 187 & Yulin & Guangxi & 547,924 & $10,728,888$ & $19,278.3$ & 116 & 349 & Huaibei & Anhui & 854,696 & $5,517,043$ & $6,355.2$ \\
\hline 29 & 103 & Zhucheng & Shandong & 586,652 & $17,611,037$ & $29,555.5$ & 73 & 188 & Xuanwei & Yunnan & 584,076 & $10,715,769$ & $18,062.9$ & 117 & 350 & Ezhou & Hubei & 607,739 & $5,508,757$ & $8,924.2$ \\
\hline 30 & 105 & Zoucheng & Shandong & 513,418 & $17,204,337$ & $32,991.4$ & 74 & 189 & Anqing & Anhui & 570,538 & $10,572,837$ & $18,244.9$ & 118 & 356 & Yanji & Jilin & 505,516 & $5,298,157$ & $10,318.7$ \\
\hline 31 & 106 & Zhuzhou & Hunan & 999,404 & $17,182,241$ & $16,926.7$ & 75 & 190 & Shaoyang & Hunan & 574,527 & $10,566,622$ & $18,107.6$ & 119 & 362 & Dandong & Liaoning & 775,787 & $5,186,987$ & $6,582.7$ \\
\hline 32 & 107 & Yuyao & Zhejiang & 672,909 & $17,170,503$ & $25,122.4$ & 76 & 192 & Ma'anshan & Anhui & 657,847 & $10,314,592$ & $15,437.0$ & 120 & 384 & Laiwu & Shandong & 666,805 & $4,852,789$ & $7,143.8$ \\
\hline 33 & 113 & Jimo & Shandong & 565,135 & $16,692,681$ & $29,080.9$ & 77 & 193 & Yiyang & Hunan & 697,607 & $10,310,449$ & $14,551.3$ & 121 & 387 & Baishan & Jilin & 503,287 & $4,832,764$ & $9,454.0$ \\
\hline 34 & 114 & Zunyi & Guizhou & 995,311 & $16,598,774$ & $16,419.2$ & 78 & 195 & Luzhou & Sichuan & 742,274 & $10,232,424$ & $13,572.2$ & 122 & 400 & Liaoyang & Liaoning & 735,047 & $4,604,901$ & $6,167.9$ \\
\hline 35 & 115 & Xianyang & Shaanxi & 835,648 & $16,509,700$ & $19,451.4$ & 79 & 205 & Shiyan & Hubei & 724,016 & $9,867,843$ & $13,418.6$ & 123 & 410 & Huludao & Liaoning & 646,482 & $4,470,255$ & $6,807.9$ \\
\hline 36 & 118 & Lianyungang & Jiangsu & 897,393 & $16,409,579$ & $18,003.2$ & 80 & 209 & Leshan & Sichuan & 678,752 & $9,712,482$ & $14,088.1$ & 124 & 422 & Yangquan & Shanxi & 623,671 & $4,301,084$ & $6,789.8$ \\
\hline 37 & 119 & Rugao & Jiangsu & 614,909 & $16,298,409$ & $26,095.7$ & 81 & 210 & Fuyang & Anhui & 780,522 & $9,680,029$ & $12,210.3$ & 125 & 434 & Tianshui & Gansu & 544,441 & $4,077,364$ & $7,373.3$ \\
\hline 38 & 120 & Suqian & Jiangsu & 783,376 & $16,234,193$ & $20,403.0$ & 82 & 213 & Qingyuan & Guangdong & 916,453 & $9,581,979$ & $10,293.9$ & 126 & 454 & Wuhai & Inner Mongolia & 502,704 & $3,865,383$ & $7,570.3$ \\
\hline 39 & 121 & Zhuji & Zhejiang & 606,683 & $15,843,374$ & $25,711.1$ & 83 & 214 & Bengbu & Anhui & 793,866 & $9,568,859$ & $11,867.2$ & 127 & 456 & Haicheng & Liaoning & 687,223 & $3,854,335$ & $5,521.9$ \\
\hline 40 & 122 & Xinghua & Jiangsu & 575,288 & $15,777,777$ & $27,001.9$ & 84 & 216 & Suzhou & Anhui & 742,685 & $9,334,092$ & $12,373.8$ & 128 & 472 & Jixi & Heilongjiang & 746,889 & $3,579,518$ & $4,718.5$ \\
\hline 41 & 123 & Huzhou & Zhejiang & 748,471 & $15,773,634$ & $20,748.7$ & 85 & 217 & Qinhuangdao & Hebei & 967,877 & $9,317,520$ & $9,478.0$ & 129 & 511 & Fuxin & Liaoning & 750,283 & $2,815,833$ & $3,695.0$ \\
\hline 42 & 124 & Luyang & Hunan & 588,081 & $15,732,205$ & $26,338.3$ & 86 & 220 & Puning & Guangdong & 874,954 & $9,137,991$ & $10,282.5$ & 130 & 576 & Hegang & Heilongjiang & 600,941 & $1,823,593$ & $2,987.7$ \\
\hline 43 & 129 & Chenzhou & Hunan & 582,971 & $15,219,168$ & $25,702.7$ & 87 & 222 & Liupanshui & Guizhou & 621,488 & $9,071,013$ & $14,370.0$ & 131 & 586 & Yichun & Heilongjiang & 694,019 & $1,734,520$ & $2,460.6$ \\
\hline 44 & 130 & Linhai & Zhejiang & 503,377 & $15,048,616$ & $29,433.2$ & 88 & 225 & Huangshi & Hubei & 691,963 & $9,015,084$ & $12,826.9$ & 132 & 606 & Qitaihe & Heilongjiang & 503,678 & $1,495,609$ & $2,923.5$ \\
\hline
\end{tabular}

Por último, en la Tabla 5 se muestra la importancia relativa del total de ciudades intermedias en la población, PIB y PIB PC agregados en cada país. Como resultado del proceso de urbanización, en 2005 89,8\% del PIB en México se generaba en zonas urbanas mayores a 15.000 habitantes y en China 35,3\%; indicando una mayor concentración económica en el país latinoamericano. Considerando las ciudades intermedias se observa que $23,9 \%$ y $19,1 \%$ del PIB nacional se producía en este tipo de área urbana en México y China, respectivamente. Esto es, en México 26,6\% del PIB urbano se obtiene en ciudades intermedias mientras que en China este dato corresponde a 23,1\%. 
Tabla 5. Ciudades intermedias en México y China

\begin{tabular}{lccccc}
\hline & Población & PIB & PIBPC & Población & PIB \\
\hline & 2010 & 2005 & 2005 & (participación \%) \\
México & $127,550,000$ & $1,210,480,781,402$ & $8,808.6$ & 100.00 & 100.00 \\
Total Ciudades & $104,565,558$ & $1,086,681,114,360$ & $7,081.4$ & 81.98 & 89.77 \\
Total Ciudades Intermedias & $21,920,405$ & $288,971,898,242$ & $13,654.9$ & 17.19 & 23.87 \\
& 2010 & 2016 & 2016 & (participación \%) \\
China & $1,339,724,852$ & $8,908,300,778,496$ & $6,496.6$ & 100.00 & 100.00 \\
Total Ciudades & $473,434,901$ & $7,393,889,646,152$ & $18,317.7$ & 35.34 & 83.00 \\
Total Ciudades Intermedias & $92,901,914$ & $1,701,123,336,415$ & $18,263.0$ & 6.93 & 19.10 \\
\hline
\end{tabular}

PIB en dólares constantes al 2016 y PIB PC al 2005.

Fuente: elaboración propia con datos de United Nations, Department of Economic and Social Affairs, Population Division (2014). World Urbanization Prospects: The 2014 Revision.

Fuente: elaboración propia con datos de United Nations, Department of Economic and Social Affairs, Population Division (2014). World Urbanization Prospects: The 2014 Revision.

No obstante, sobresalen dos aspectos centrales: primero, la participación del PIB de ciudades intermedias en el PIB nacional es mayor que el peso de su población en el total nacional; sugiriendo una posición más central que la indicada por la población, dada la actividad económica. Segundo, y en línea con esto, el PIB per cápita obtenido en las ciudades intermedias es mayor que el promedio para el total de ciudades; indicando un nivel de vida promedio más alto que la media nacional.

\section{Metodología y datos}

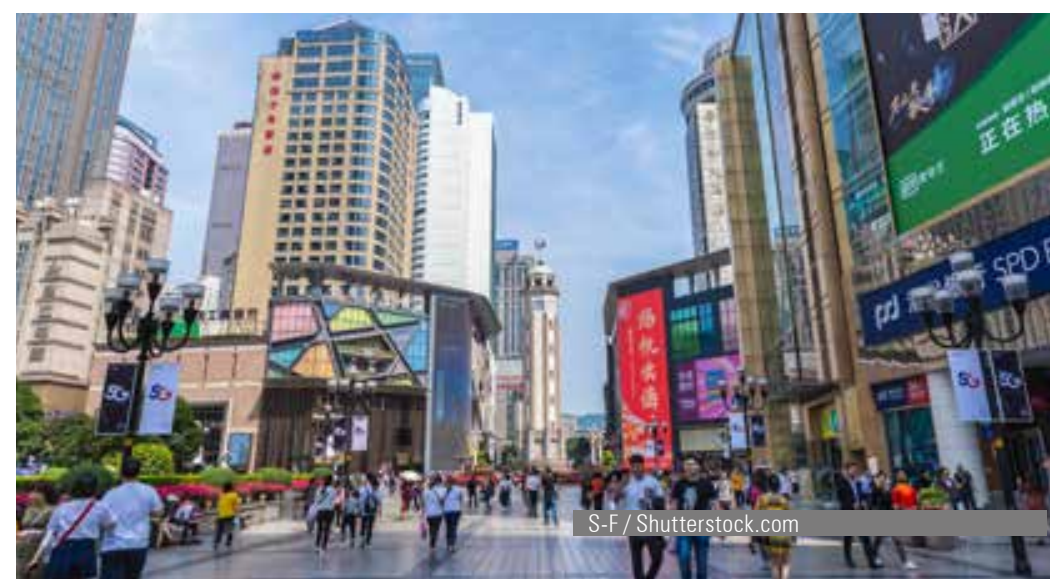

Una importante consecuencia de la urbanización es el cambio en la distribución del tamaño de las ciudades. El objetivo de este estudio es investigar esta distribución. La forma de la distribución del tamaño de las ciudades fue propuesta inicialmente por Auerbach (1913), basado en la distribución de Pareto: $y=A x^{-\alpha}$, donde $x$ es la población de la ciudad; $y$ el rango de la ciudad, una vez que estas se orden de mayor a menor tamaño; $A$ una constante positiva; y $\alpha$ el exponente de Pareto. Una forma de estimar $\alpha$ es mediante una regresión por MCO de los logaritmos rango-tamaño: $\log y=\log A-\alpha \log x$; por ende, $\alpha(\alpha>0)$ es una medida de la concentración de la población entre ciudades de diferentes tamaños. 
Sin embargo, Gabaix y loannides (2004) argumentan que las estimaciones por Mínimos Cuadrados Ordinarios son sesgadas en muestras pequeñas, como puede ser estimado este caso, y que llevaría a considerar a las mayores ciudades más grandes de lo que en realidad son. En concreto, este método subestima el valor verdadero del error estándar del exponente de Pareto, lo que conduce al rechazo del exponente. Por ende, proponen como solución simple corregir el sesgo mediante un cambio de $1 / 2$ para que el rango sea óptimo. En este sentido, el modelo formal se define como:

$$
\log (y-1 / 2)=\alpha \operatorname{olog} A-\alpha 1^{\prime} \log x
$$

Adicionalmente, Gabaix y Ibragimov (2012) muestran que bajo esta especificación el error estándar se distribuye asintóticamente como â' (2/n)1/2, donde $n$ es el tamaño de la muestra. La ecuación (2) se estima por separado para México y China empleando MCO con errores estándar robustos que se prueban con el test de heteroscedasticidad de Cook-Weisberg.

Por último, para verificar la validez de la Ley de Zipf debe considerarse que la regla rango-tamaño representa esta ley cuando se considera únicamente a las grandes ciudades, pero esto no sucede necesariamente si se incluyen todas las ciudades que, en función de su definición, puede incluir localidades con al menos 2.500 habitantes. En este documento se considera como ciudad a la aglomeración urbana con al menos 15.000 habitantes. Para Fazio y Modica (2015) la razón de la alta sensibilidad de $\alpha$ es producto de la inclusión de toda la gama de ciudades. Además, Cristelli et al. (2012) argumentan que el incumplimiento de la Ley de Zipf se debe a que esta tiene una forma funcional dependiente del número de eventos $N$, por ende, requiere del cumplimiento de la propiedad de "coherencia". Es decir, como en las submuestras los elementos faltantes llevan inevitablemente a desviaciones de la Ley de Zipf, lo que ocurre especialmente con subconjuntos que incluyen únicamente a las ciudades más grandes, así que debe considerarse ampliaciones de las muestras para incorporar el mayor $N$ posible.

En consecuencia, se emplea un tipo de regresión útil cuando la variable dependiente es muy sensible a la variable independiente en diferentes segmentos. En particular, se sigue el método de regresión de rangos móviles, en el que se repite el proceso Mínimos Cuadrados Ordinarios, pero usando un punto de truncamiento menor que es, al mismo tiempo, el punto de partida para la siguiente submuestra. Este punto es el mismo y se fija en la ciudad más grande. El punto de truncamiento inferior disminuye en una ciudad con cada submuestra, por lo que el tamaño de esta aumenta en uno. De este modo, se establece una submuestra para estimar con tamaño arbitrario de $\check{n} 1=20$, es decir, las primeras veinte ciudades con mayor PIB. La segunda submuestra es $\check{n}_{2}=\check{n}_{1}+1=21$, que incluye a las veintiún ciudades más grandes; la tercera submuestra es $\check{n}_{3}=\check{n}_{2}+1=22$, con las veintidós mayores ciudades. El proceso continúa hasta incluir en la submuestra a la ciudad más pequeña, por lo que esta representa la muestra total.

De acuerdo con datos oficiales de cada país, se observa una mayor dispersión de la población que habita en ciudades de al menos 15.000 habitantes en México en comparación con China. Esto lleva a que la muestra completa para el primer país sea de 1.116 ciudades con al menos 15.000 habitantes en 2005, que se ordenan de forma descendiente, por lo que Guadalajara (Jalisco) es la ciudad de mayor tamaño y Texistepec (Veracruz) la menor. Para China la muestra total es de 639 ciudades en 2016 con al menos 15.000 habi- 
tantes, con Shanghai (Shanghai) como la ciudad de mayor tamaño y Nyingchi (Tibet) la más pequeña. Con este método se estiman 1.097 $(=1.116-20+1)$ coeficientes de Pareto al considerar ese número de regresiones para México y $620(=639-20+1)$ coeficientes para China.

La fuente de información es el Instituto Nacional de Estadística, Geografía e Informática (INEGI) para México y el National Bureau of Statistics para China (NBS). Dada la especificidad del análisis, los datos son muy limitados y para periodos distintos en cada país. Así, para México se obtienen datos de la población por ciudad para el 2010 y del PIB para el 2005; para China la información de la población es para el año 2010 y del PIB para 2016. Por esta razón, las comparaciones entre países deben tomarse con cautela.

\section{Resultados y discusión}

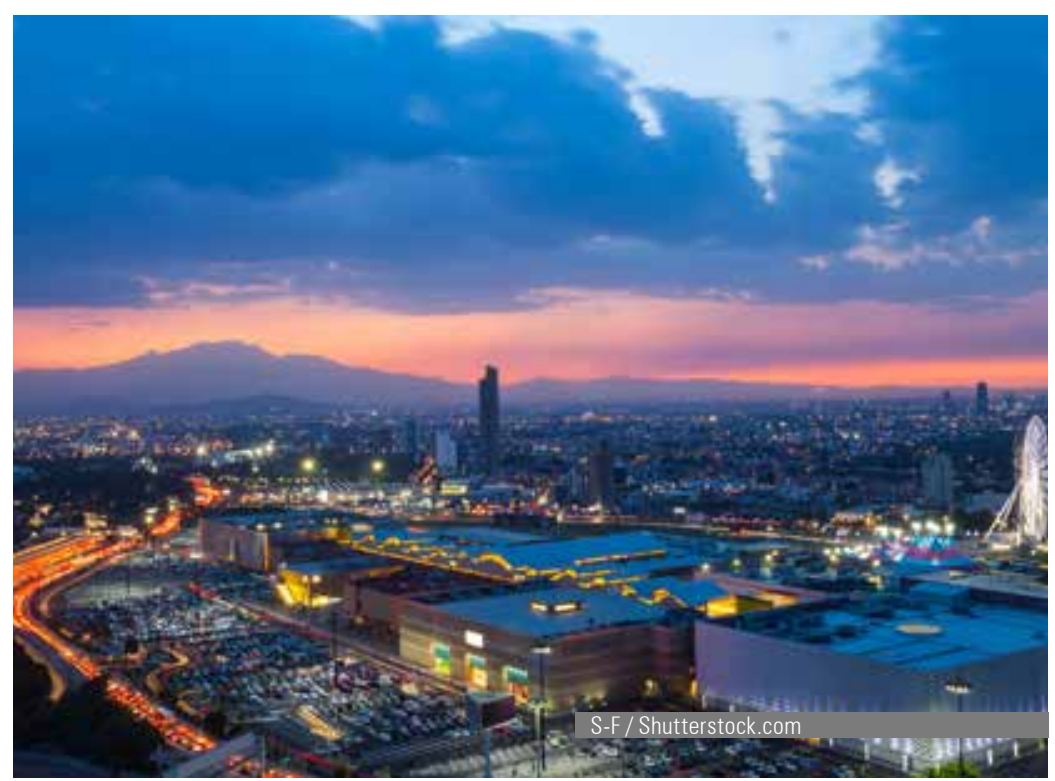

Los resultados de las estimaciones de las ecuaciones (1) y (2) se presentan en la Tabla 6. Aunque las estimaciones de las dos especificaciones tienen un comportamiento similar en el que se aprecia una disminución de $\alpha$ y $\alpha^{\prime}$ en ambos países, existen diferencias sutiles. Las estimaciones para México son en todos los casos menores que para China, salvo para las muestras de 600 ciudades o más. Sin embargo, los coeficientes para la muestra total de México $\left(\alpha\right.$ y $\left.\alpha^{\prime}\right)$ son mayores a los chinos. También, como se esperaba, para los dos países siempre se mantiene $\alpha<\alpha^{\prime}$.

Para el caso de México, se tiene que el valor promedio del exponente de Pareto $(\alpha)$ para todas las regresiones es cercano a 0,661 , mientras que el valor de este exponente para la muestra completa (1.116 ciudades) es 0,562. Para el exponente $\alpha^{\prime}$ los valores respectivos son 0,769 y 0,596. Así, todos los exponentes estimados por (1) son inferiores a uno y solo cinco son mayores a la unidad cuando se estiman por (2). En general, se tiene que la Ley de Zipf parece no cumplirse en las ciudades mexicanas considerando su tamaño económico. Resultado similar se encuentra en Soo (2005) y Pérez et al. (2015) quienes estiman coeficientes mayores a la unidad. 
En concreto, cuando se consideran las submuestras estimadas con entre 20 y 40 ciudades, los exponentes de Pareto ajustados $\left(\alpha^{\prime}\right)$ son 1,133 y 1,024 , respectivamente. Esto indica que la ciudad más grande (Guadalajara, Jalis$\mathrm{co}$ ), esto es, la de mayor PIB, tiene mayor peso relativo que lo señalado por la Ley de Zipf. Esto se entiende como una mayor desigualdad en el tamaño económico de las ciudades. Sin embargo, la desigualdad es menor conforme las submuestras aumentan de tamaño; el coeficiente de Pareto estimado se reduce por debajo de la unidad. De hecho, el valor estimado de $\alpha^{\prime}$ es monótonamente decreciente para todas las submuestras. Así, desde que el punto de truncamiento es cincuenta ciudades, el exponente muestra un valor menor a la unidad, lo que implica una distribución más homogénea del tamaño económico de estas urbes, existiendo, a partir de este punto, un cierto equilibrio entre el poder de las mismas.

Tabla 6. Coeficiente de Pareto. Regresiones de rangos móviles

\begin{tabular}{|c|c|c|c|c|c|c|c|c|c|c|c|c|c|c|c|c|c|c|c|}
\hline \multicolumn{7}{|c|}{ México (2005) } & \multicolumn{13}{|c|}{ China (2010) } \\
\hline$n$ & $\alpha$ & & $e e$ & $\alpha^{\prime}$ & $e e$ & $n c i$ & $n$ & $\alpha$ & $e e$ & $\alpha^{\prime}$ & & $e e$ & $n c i$ & $n$ & $\alpha$ & $e e$ & $\alpha^{\prime}$ & $e e$ & $n c i$ \\
\hline 20 & 0.9323 & ${ }^{* * *}$ & 0.2948 & $1.1326^{* * *}$ & 0.3582 & 9 & 20 & $1.4785^{* * *}$ & 0.4675 & 1.9770 & ${ }^{* * *}$ & 0.6252 & 0 & 260 & $0.8845^{* *}$ & 0.0872 & $0.9147^{* *}$ & 0.0901 & 98 \\
\hline 25 & 0.9202 & ${ }^{* * *}$ & 0.2603 & $1.1045^{* * *}$ & 0.3124 & 13 & 40 & $1.3692^{* * *}$ & 0.3062 & 1.8417 & ${ }^{* * *}$ & 0.4118 & 1 & 280 & $0.8428^{* *}$ & 0.0800 & $0.8807^{* *}$ & 0.0836 & 102 \\
\hline 30 & 0.9103 & ${ }^{* * *}$ & 0.2350 & $1.0798^{* * *}$ & 0.2788 & 17 & 50 & $1.2779^{* * *}$ & 0.2556 & 1.8290 & ${ }^{* * *}$ & 0.3658 & 6 & 300 & $0.8157^{* *}$ & 0.0748 & $0.8557^{* *}$ & 0.0785 & 109 \\
\hline 35 & 0.8883 & ${ }^{* * *}$ & 0.2123 & $1.0493^{* * *}$ & 0.2508 & 20 & 60 & $1.4643^{* * *}$ & 0.2673 & 1.6872 & ${ }^{* * *}$ & 0.3080 & 10 & 350 & $0.8043^{* *}$ & 0.0683 & $0.8453^{* *}$ & 0.0718 & 117 \\
\hline 40 & 0.7839 & $* * *$ & 0.1753 & $1.0240^{* * *}$ & 0.2290 & 23 & 70 & $1.3490^{* * *}$ & 0.2280 & 1.6068 & $* * *$ & 0.2716 & 14 & 400 & $0.7995^{* *}$ & 0.0635 & $0.8296^{* *}$ & 0.0659 & 122 \\
\hline 50 & 0.7632 & $* * *$ & 0.1526 & $0.9876^{* * *}$ & 0.1975 & 29 & 80 & $1.2682^{* * *}$ & 0.2005 & 1.4635 & $* * *$ & 0.2314 & 18 & 450 & $0.7391^{* *}$ & 0.0554 & $0.7873^{* *}$ & 0.0590 & 125 \\
\hline 60 & 0.7544 & $* * *$ & 0.1377 & $0.9429^{* * *}$ & 0.1721 & 31 & 90 & $1.0921^{* * *}$ & 0.1628 & 1.3502 & $* * *$ & 0.2013 & 22 & 500 & $0.6781^{*}$ & 0.0482 & $0.6868^{* *}$ & 0.0488 & 128 \\
\hline 70 & 0.7391 & $* * *$ & 0.1249 & $0.9146^{* * *}$ & 0.1546 & 32 & 100 & $1.0571^{* * *}$ & 0.1495 & 1.2294 & $* * *$ & 0.1739 & 27 & 600 & 0.4982 & 0.0323 & 0.5353 & 0.0347 & 131 \\
\hline 100 & 0.7197 & ${ }^{* * *}$ & 0.1018 & $0.8668^{* * *}$ & 0.1226 & 32 & 110 & $1.0082^{* * *}$ & 0.1359 & 1.1503 & $* * *$ & 0.1551 & 32 & Total & 0.4891 & 0.0288 & 0.5494 & 0.0324 & 132 \\
\hline 200 & 0.5928 & ${ }^{* *}$ & 0.0593 & $0.6867^{* *}$ & 0.0687 & 32 & 120 & $0.9943^{* * *}$ & 0.1284 & 1.1368 & $* * *$ & 0.1468 & 38 & Promedio & 0.9770 & - & 1.1397 & - & 132 \\
\hline 300 & 0.5866 & ${ }^{*}$ & 0.0479 & $0.6018^{*}$ & 0.0491 & 32 & 130 & $0.9812^{* * *}$ & 0.1217 & 1.1105 & $* * *$ & 0.1377 & 44 & & & & & & \\
\hline 400 & 0.5623 & & 0.0398 & 0.5834 & 0.0413 & 32 & 140 & $0.9685^{* * *}$ & 0.1158 & 1.1094 & ${ }^{* * *}$ & 0.1326 & 51 & & & & & & \\
\hline 500 & 0.5425 & & 0.0343 & 0.5635 & 0.0356 & 32 & 150 & $0.9605^{* * *}$ & 0.1109 & 1.1034 & $* * *$ & 0.1274 & 56 & & & & & & \\
\hline 600 & 0.5227 & & 0.0302 & 0.5438 & 0.0314 & 32 & 160 & $0.9546^{* * *}$ & 0.1067 & 1.1005 & $* * *$ & 0.1230 & 62 & & & & & & \\
\hline 700 & 0.5122 & & 0.0274 & 0.5346 & 0.0286 & 32 & 170 & $0.9535^{* * *}$ & 0.1034 & 1.0998 & $* * *$ & 0.1193 & 65 & & & & & & \\
\hline 800 & 0.4957 & & 0.0248 & 0.5167 & 0.0258 & 32 & 180 & $0.9513^{* * *}$ & 0.1003 & 1.0993 & $* * *$ & 0.1159 & 69 & & & & & & \\
\hline 900 & 0.4676 & & 0.0220 & 0.5076 & 0.0239 & 32 & 190 & $0.9480^{* * *}$ & 0.0973 & 1.0991 & $* * *$ & 0.1128 & 75 & & & & & & \\
\hline 1000 & 0.4526 & & 0.0202 & 0.4855 & 0.0217 & 32 & 200 & $0.9467^{* * *}$ & 0.0947 & 1.0986 & $* * *$ & 0.1099 & 78 & & & & & & \\
\hline Total & 0.5618 & & 0.0238 & 0.5958 & 0.0252 & 32 & 220 & $0.9325^{* *}$ & 0.0889 & 1.0817 & $* * *$ & 0.1031 & 86 & & & & & & \\
\hline Prom & 0.6613 & & - & 0.7691 & - & 32 & 240 & $0.8973^{* *}$ & 0.0819 & 1.0534 & $* *$ & 0.0962 & 94 & & & & & & \\
\hline
\end{tabular}

Fuente: elaboración propia.

Los errores estándar (columnas 4, 7, 12, 15, 20 y 23 en la Tabla 6) se corrigen por el método propuesto por Gabaix y lbragimov (2012) y pasan el test de heteroscedasticidad de Cook-Weisberg. Las estimaciones son significativas hasta la submuestra de tamaño 300 que incluye las 32 ciudades intermedias. En esta, se registra una distribución relativamente igual del poder económico de las ciudades; en contraste, en la primera submuestra (20 ciudades), el $\alpha^{\prime}>1$ e incluye nueve ciudades intermedias. Aún más, en la submuestra de mayor tamaño con $\alpha_{1}>1(n=40)$ se incluyen 23 ciudades intermedias. Por el contrario, cuando se considera la submuestra $n=50$, que registra un $\alpha_{1}<1$, se tendría que 90,6\% de las ciudades intermedias están dentro de un sistema urbano con una distribución del tamaño económico equitativa. Por ende, es posible aceptar que el poder económico del conjunto de las ciudades intermedias es relativamente equitativo al de las ciudades de mayor tamaño en la submuestra. 
Aparte, en el caso de China, se tiene que el valor promedio del exponente de Pareto $(\alpha)$ para todas las regresiones es cercano a 0,977 , mientras que el valor de este exponente para la muestra completa (632 ciudades) es 0,489. Para el exponente $\alpha^{\prime}$ los valores respectivos son 0,549 y 1,139. En contraste a los resultados para México, se estiman más exponentes por (1) o (2) superiores a uno, señalando un mayor desequilibrio en el sistema urbano de esa economía asiática. Esta situación se cumple para la submuestra de las primeras 200 ciudades de mayor tamaño económico. En cualquier caso, se tiene que la Ley de Zipf tampoco perece cumplirse en las ciudades chinas considerando su tamaño económico. Este resultado es similar al de Soo (2005) y Xu y Zhu (2009).

En particular, cuando las submuestras estimadas incluyen un número de ciudades entre 20 y 200 aglomeraciones, los exponentes de Pareto son 1,977 y 1,098 de acuerdo con (2), respectivamente, para un valor promedio del coeficiente de 1,139; lo que indica que la ciudad más grande (Shanghai, Shanghai), es decir, la de mayor PIB, tiene menor peso que lo señalado por la Ley de Zipf, esto es, la desigualdad en el tamaño económico de las ciudades es menor.

De cualquier forma, esta situación disminuye conforme las submuestras aumentan de tamaño, reduciéndose el coeficiente estimado de Pareto por debajo de la unidad. De nuevo, el valor estimado de $\alpha^{\prime}$ es monótonamente decreciente para todas las submuestras. Se observa que desde que el punto de truncamiento es 240 ciudades, el exponente muestra un valor menor a la unidad, lo que implica una distribución más homogénea del tamaño económico de las ciudades, existiendo en estas submuestras un cierto equilibrio entre el poder de las ciudades. Sin embargo, el rango de tamaño con $\alpha^{\prime}>1$ agrupa 59,1\% del total de ciudades intermedias — las de mayor PIB de este grupo-, por lo que la contribución al desempeño económico de las principales aglomeraciones intermedias se genera en las submuestras más pequeñas caracterizadas por una distribución menos equitativa, con predominio de pocas ciudades. Esto contrasta con el caso mexicano, por lo que parece existir mayor peso relativo de las ciudades intermedias en México que en China.

Los errores estándar (columnas 9, 11, 15 y 17 en la Tabla 6) se corrigen por el método propuesto por Gabaix y lbragimov (2012) y pasan el test de heteroscedasticidad de Cook-Weisberg. Las estimaciones son significativas hasta la submuestra de tamaño 500 que incluye 128 de las 132 ciudades intermedias. En esta, se registra una distribución relativamente igual del poder económico de las ciudades; en contraste, en la primera submuestra (20 ciudades), el $\alpha^{\prime}>1$ que no incluye ciudades intermedias. Aún más, en la submuestra de mayor tamaño con $\alpha_{1}>1 \quad(n=200)$ se incluyen 78 ciudades intermedias y si se considera la submuestra $n=240$, también con $\alpha_{1}>1$, se tendría que $65,1 \%$ de las ciudades intermedias están dentro de un sistema urbano donde parece existir una sobrerrepresentación de las ciudades de mayor tamaño económico. En cualquier caso, cuando se considera la submuestra $n=500$, que registra un $\alpha_{1}<1$, se tendría que $96,9 \%$ de las ciudades intermedias están dentro de un sistema urbano con una distribución del tamaño económico equitativa. Por ende, es posible aceptar que el poder económico del conjunto de ciudades intermedias es relativamente equitativo al de las ciudades de mayor tamaño en la submuestra. 
Cabe señalar que para las submuestras de mayor tamaño el valor del exponente de Pareto disminuye. De hecho, para México desde la submuestra de tamaño 200 y para China desde la submuestra $(n=500)$ se tiene un grado de dispersión excesivo $\left(\alpha_{1}<0.7\right)$, de acuerdo con lo señalado por Brañas y Alcalá (2014).

La relevancia de las ciudades intermedias puede apreciarse gráficamente. La Figura 1, paneles a y b, muestra la dispersión del logaritmo del rango contra el logaritmo del tamaño económico (PIB) de las ciudades para la muestra completa en ambos países. El diagrama no refleja una línea recta sino cóncava, lo que está de acuerdo con el hecho de que el exponente de Pareto varía en función del tamaño de la submuestra. La concavidad sugiere que las ciudades más grandes son demasiado pequeñas y las ciudades de menor tamaño están subrepresentadas. Rossi y Wright (2007) enfatizan esta regularidad empírica. La concavidad se registra en los dos países, por lo que ambos se desvían de la Ley de Zipf, lo que es evidencia de distorsiones del sistema urbano, consecuencia, posiblemente, de aglomeraciones de menor tamaño con mayor contribución a la actividad económica, si bien, con menores niveles de eficiencia por representar menores economías de urbanización asociadas con cuestiones institucionales, de localización e históricas (Soo, 2005).

Por último, considerando la heterogeneidad en el tamaño de las economías y de las aglomeraciones en ambos países, se acepta que el efecto de las ciudades intermedias en el desempeño económico es mayor en México que en China, puesto que en México las submuestras con $\alpha^{\prime}<1$ (distribución más equitativa, sin predominio relevante de una ciudad) incluye una mayor participación de ciudades intermedias que en China.

\section{Figura 1. Logaritmo del tamaño vis-á-vis logaritmo del rango, muestra completa}

(a) México

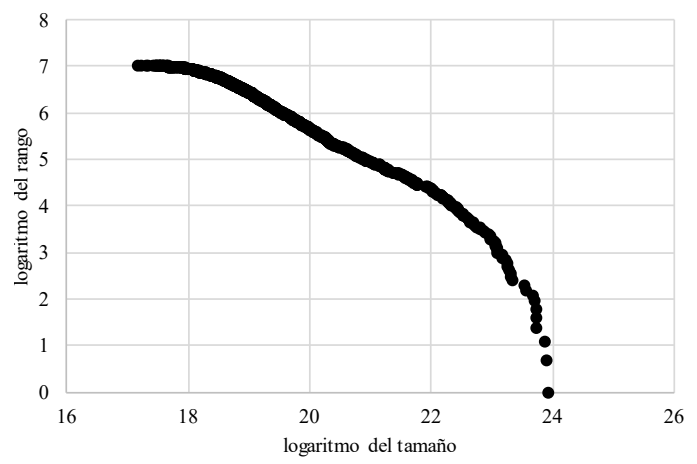

(b) China

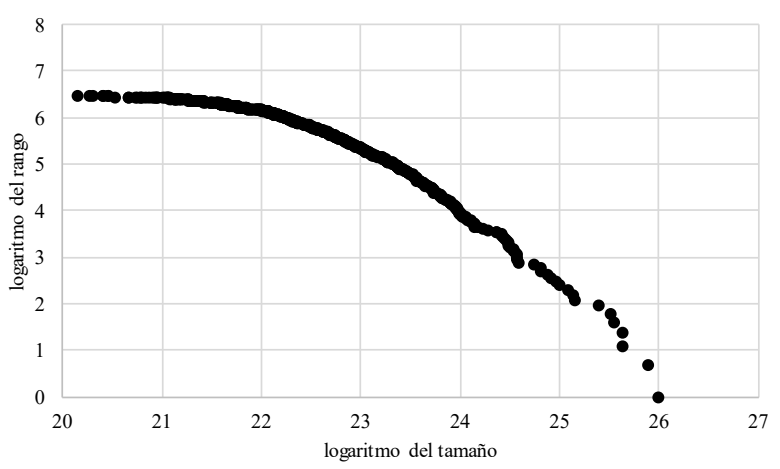

Fuente: elaboración propia. 


\section{Reflexiones finales}

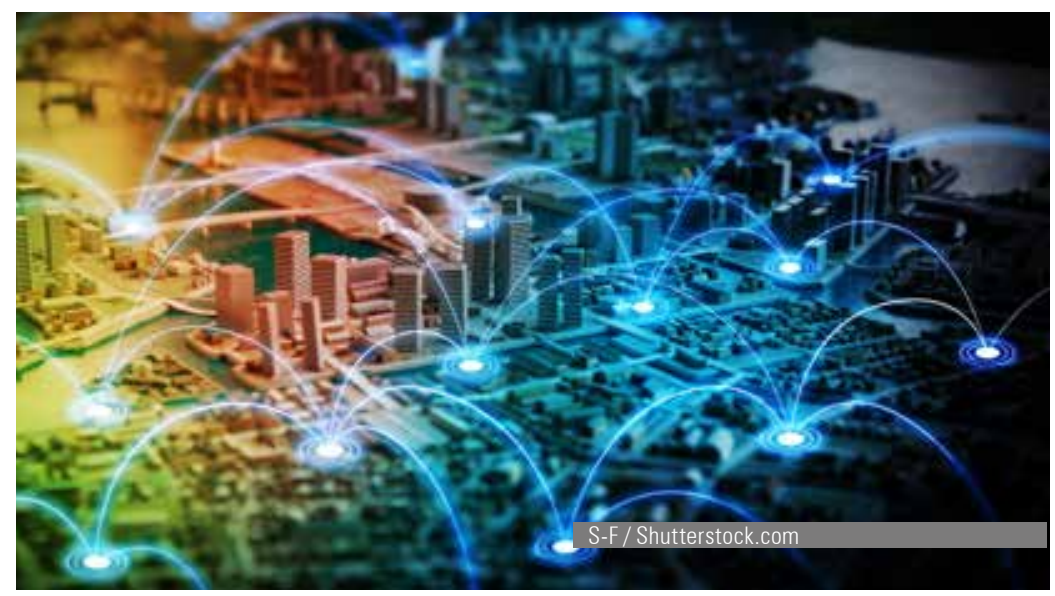

El empleo de la Ley de Pareto y la Ley de Zipf mediante el ajuste en la estimación MCO permite analizar los sistemas urbanos de México y China. Los resultados muestran que el exponente de Pareto es sensible al tamaño de la muestra y al punto de truncamiento de acuerdo al método de regresiones de rangos móviles.

En general, se tiene que no se cumple la Ley de Zipf identificada por Gabaix (1999). No obstante, las estimaciones por submuestras sugieren que existe una distribución del tamaño de las ciudades relativamente equitativa en ambas naciones, lo que permite aceptar la hipótesis de un coeficiente de Pareto menor a la unidad. Como las principales ciudades intermedias se agrupan en sistemas urbanos estimados como equitativos, se acepta que esta desviación del exponente de la Ley de Zipf se explica por el mayor peso económico relativo de las ciudades intermedias.

Al respecto, la desviación del coeficiente de Pareto de la unidad (por debajo de uno), que implica sistemas urbanos más equitativos, es presumiblemente consecuencia de alguna distorsión en las decisiones de los individuos que migran hacia ciudades intermedias, incluidas en este rango, al percibir mayores oportunidades económicas, producto de choques externos o de intervenciones gubernamentales que impulsan estas regiones por encima de otras de mayor tamaño — por arriba en el ranking —. Estas distorsiones generan que ciudades de menor rango tengan mayor peso en el desempeño económico agregado — PIB —, compensando en algún grado el menor dinamismo de las ciudades más grandes, sin que estas últimas muestren algún predominio sobre las intermedias, situación que se presenta en mayor medida en el caso de la economía mexicana.

Por último, la distribución del tamaño de las ciudades relativamente equitativa que se encontró en ambos casos tiende a favorecer el desempeño económico de un país. Ceteris paribus, las ciudades intermedias parecen tener una capacidad de producción con más posibilidades de expansión en comparación a las ciudades de mayor tamaño económico que registran una elevada población y capacidad de producción cercana al punto de saturación. Lo anterior implicaría que sin esta distribución de ciudades la dinámica económica de ambas naciones registraría menores estándares a los actuales, con efectos directos en el desarrollo. 
Por ende, los hacedores de política deben considerar que estas ciudades representan una fuerza motora fundamental y establecer políticas regionales y urbanas que incentiven su desarrollo económico. Así, una política particular puede fomentar los lazos económicos y sociales entre ciudades intermedias en la misma región o regiones conexas, y entre estas y ciudades de menor tamaño dentro del área de influencia de las primeras, que permitan consolidar su posición productiva en el sistema urbano nacional y regional.

\section{Referencias}

- Arshad, S., Hu, S. Ashraf, B. (2018). Zipf's law and city size distribution: A survey of the literatura and future research agenda. Physica A, 492:75-92.

- Berry, B. y Okulicz, A. (2012). The City Size Distribution Debate: Resolution For US Urban Regions and Megalopolitan Areas. Cities, 29(1):17-23.

- Brañas, P. y Alcalá, F. (2014). Entropía, aglomeración urbana y la Ley del "1": Evidencia para las regiones españolas. XVII Encuentro de Economía Aplicada, Gran Canaria.

- Cristelli, M.; Batty, M. y Pietronero, L. (2012). There is More Than a Power Law in Zipf. Scientific Reports, 2:812.

- Fang, L., Li, P.y Song, S. (2017). China's Development Policies and City Size Distribution: An Analysis Based on Zipf'S Law. Urban Studies, 52(2):2818-2834.

- Fazio, G. y Modica, M. (2015). Pareto or Log-Normal? Best Fit and Truncation in the Distribution of All Cities. Journal of Regional Science, 55(5):736-756.

- Gabaix, X. (1999). Zipf's Law for Cities: An Explanation. Quarterly Journal of Economics, 114:739-767.

- Gabaix, X. y loannides, Y. (2004). The Evolution of City Size Distribution. Handbook of Regional and Urban Economics, 4:2431-2378.

- Gabaix, X. e lbragimov, R. (2012). Rank-1/2: A Simple Way to Improve the OLS Estimation of Tail Exponents. Journal of Business and Economic Statistics, 29(1):24-39.

- Hofmann, A. y Wan, G. (2013). Determinants of urbanization. Asian Devolopment Bank: Manila.

- Krugman, P. (1996). The self-organizing economy. Blackwell: Cambridge.

- Lin, C., Cheng, M. y Yang, C. (2013). Zipf's Law and City Size Distribution in China, Revista de Humanidades y Ciencias Sociales, 25(3) :525-550.

- ONU-Habitat. (2012). Estado de las ciudades de América Latina y El Caribe 2012. Rumbo a una nueva transición urbana. ONU-Habitat: Río de Janeiro.

- Parr, J. y Jones, C. (1983). City Size Distributions and Urban Density Functions: Some Interrelationships. Journal of Regional Science, 23(3):283-307.

- Peng, G. (2010). Zipf's Law for Chinese Cities: Rolling Sample Regressions, Physica A, 389:3804-3813.

- Pérez, E., Guzmán, L. y Angulo, F. (2015). Distributions of City Sizes in Mexico During the 20th Century. Chaos, Solutions \& Fractals, 73:64-70.

- Rani, C. y Tripathi, S. (2016). Determinants of Urbanization in Different Size/Class Distribution of Cities/ Towns in India. MPRA74757.

- Rosen, K. y Resnick, M. (1980). The Size Distribution of Cities: An Examination of the Pareto Law and Primacy. Journal of Urban Economics, 8(2):165-186.

- Rossi, E. y Wright, M. (2007). Urban Structure and Growth. Review of Economic Studies, 74(2):597-624.

- Sobrino, J. (1999). Desarrollo urbano en México a partir de 1980. El Colegio Mexiquense: Toluca.

- Soo, K. (2005). Zipf's Law for Cities: A Cross-Country Investigation. Regional Science and Urban Economics, 35:239-263.

- Veneri, P. (2016). City Size Distribution Across the OECD: Does the Definition of Cities Matter? Computers, Environment and Urban Systems, 59:86-94.

- Xu, Z. y Zhu, N. (2009). City Size Distribution in China: Are Large Cities Dominant? Urban Studies, $46(10): 2159-2185$. 